\title{
Biliary peritonitis secondary to perforation of common bile duct: an unusual presentation of chronic calcific pancreatitis
}

\author{
Arvind Kumar, R. Kataria, T.K. Chattopadhyay, P.K. Karak ${ }^{1}$ and \\ R.K. Tandon ${ }^{2}$
}

Departments of Surgery, ${ }^{1}$ Radiodiagnosis and ${ }^{2}$ Gastroenterology, All India Institute of Medical Sciences, New Delhi-110029, India

\begin{abstract}
Summary: Common bile duct perforation causing biliary peritonitis is an unusual entity and a pancreatic calculus causing this perforation is all the more rare, and to our knowledge has not been reported previously. Such an unusual presentation of chronic calcific pancreatitis is herein reported.
\end{abstract}

\section{Introduction}

Cholestasis is said to complicate non-gallstone chronic pancreatitis in $3-25 \%$ of patients. It may be transient, probably the result of oedema of the pancreas with compression of the bile duct in its intra-pancreatic course, or persistent, usually occurring in severe destructive chronic pancreatitis due to cicatrization of the lower common bile duct within the head of the pancreas. We recently managed a patient with a non-gallstone chronic calcific pancreatitis presenting as biliary peritonitis due to perforation of the common bile duct. Perforation of the common bile duct due to its obstruction by a pancreatic calculi has not to our knowledge previously been reported. The clinical details of the case and a review of the relevant literature are presented here.

\section{Case report}

A 13 year old boy, presented in December 1990 with colicky, central abdominal pain for 2 weeks and abdominal distension and constipation for 5 days. He gave no history of fever, vomiting or jaundice. Examination revealed a thin-built, sicklooking, undernourished boy with low grade fever. The abdomen was distended with evidence of free fluid and generalized tenderness with guarding and rebound tenderness on the right side. Bowel sounds were sluggish; rectal examination revealed bogginess in the recto-vesical pouch. Investigations revealed a haemoglobin of $12 \mathrm{~g} / \mathrm{dl}$, white blood

Correspondence: A. Kumar, M.S.

Accepted: 2 March 1992 cells $11.2 \times 10^{6} / 1$ with polymorphonuclear leucocytosis, erythrocyte sedimentation rate $58 \mathrm{~mm}$ in the first hour. Serum bilirubin, alkaline phosphatase, proteins, transaminases and amylase were all within normal limits. Blood culture was sterile. A plain film of the abdomen showed evidence of ileus with calcification in the region of the pancreas. Ultrasound examination of the abdomen revealed ascites only. Ascitic tap showed bilious fluid.

With a diagnosis of biliary peritonitis, the patient was taken up for exploratory laparotomy after achieving fluid and electrolyte homeostasis. A litre of bilious peritoneal fluid was drained. There were fibrinous flakes over the serosa of bowel but no evidence of perforation. The gallbladder wall was oedematous but intact. It contained no calculi. The common bile duct was dilated $(1.2 \mathrm{~cm})$ with a $2 \mathrm{~cm}$ long perforation in the supraduodenal part with necrotic edges. After mobilization of the second part of the duodenum, a stone was palpated at the distal end of the common bile duct. The pancreatic head felt normal.

Cholecystectomy, choledocholithotomy, closure of the common bile duct over a T-tube and thorough peritoneal toilet was done and the abdomen closed after putting multiple tube drains. The patient had an uneventful postoperative recovery. The calculus was sent for biochemical analysis.

Three weeks postoperatively, a T-tube cholangiogram revealed a normal calibre common bile duct with free flow of contrast into the duodenum. However, contrast fluid also refluxed into the pancreatic duct. This was dilated $8 \mathrm{~mm}$ and showed filling defects suggestive of pancreatic calculi (Figure 1). Abdominal computed tomographic 
Figure 1 T-tube cholangiogram showing normal common bile duct (hollow arrow), free flow of contrast into the duodenum and reflux filling of dilated main pancreatic duct with filling defect (long arrow). Calcification seen in pancreatic tail area (small arrows).

(CT) scan revealed atrophy of the pancreatic parenchyma with calcification in the region of the body and tail. His hepatic and pancreatic function tests were within normal limits.

The biochemical analysis of the stone revealed predominantly calcium carbonate stone. No cholesterol or bile pigments were detected.

The final diagnosis therefore was non-alcoholic chronic calcific pancreatitis with an impacted pancreatic calculus at the distal end of the common bile duct causing ductal perforation and biliary peritonitis. One year later the patient is asymptomatic and his liver and pancreatic function tests remain within normal limits.

\section{Discussion}

Biliary peritonitis is a relatively uncommon condition-often neglected and uniformly fatal if left undrained. Although rupture of a pathological gallbladder, or biliary ducts or cholangitic abscess secondary to obstruction of biliary tree are known causes, rupture of the common bile duct following impaction of a pancreatic calculus in the distal common bile duct has not to our knowledge been reported to date.

Cholestasis is said to complicate non-gallstone chronic pancreatitis in $3-25 \%$ of patients. ${ }^{1}$ This may be transient, due to oedema of the pancreas with compression of common bile duct in its intra-pancreatic course, or persistent, usually associated with severe chronic pancreatitis and is due to stenosis of the lower common bile duct within the head of the pancreas. This stenosis has been attributed to peri-pancreatic sclerosis and oedema. ${ }^{2}$ The length of the stenosed segment and its clinical consequences are variable. Above the stenosis, while the common bile duct may be normal, it is generally moderately and exceptionally massively dilated resulting in cystic dilatation of the bile duct. The gallbladder and cystic duct may be distended but usually to a much lesser extent. ${ }^{3}$ The typical radiological feature in such cases is long segment stenosis of the intra- or retro-pancreatic part of the common bile duct. In non-operated cases, cholangitis is rare as the cholestasis is often mild and transient. However, after surgery and especially biliary anastomosis, it is more frequently described. ${ }^{3}$ Cholangitic abcesses and secondary biliary cirrhosis are exceptionally seen. ${ }^{2}$

The treatment of cholestasis due to nongallstone chronic pancreatitis should be initially conservative. Patients are kept under close followup. Liver function tests are performed regularly and when indicated, radiological investigations and liver biopsy may be performed. Protracted jaundice, acute cholangitis and development of secondary biliary cirrhosis are indications for surgical intervention which is usually in the form of bilio-enteric bypass. ${ }^{4}$

It is interesting that in our patient, there was no history of jaundice preceding the presentation, no were any of the liver function tests deranged on admission. At surgery, the common bile duct was found to be dilated with perforation in its supraduodenal part and a stone with biochemical features of non-biliary, pancreatic origin was extracted from its lower end. He had unequivocal evidence of chronic calcific pancreatitis of the tropics as seen in $T$-tube cholangiogram and abdominal computed tomographic (CT) scan Chronic calcific pancreatitis of the tropics is seen commonly in our country, ${ }^{5}$ though its true prevalence is not known in the absence of adequate field studies. The exact cause of this condition is not known but is presumed to be nutritional. ${ }^{6}$ Plain $\mathrm{X}$-ray, ultrasound, CT scan and endoscopic retrograde cholangiopancreatography in these cases show pancreatic calcification and dilatation of the main pancreatic duct with calculi.

In our patient, the pancreatic calculus could have reached the common bile duct after having been lodged in the common channel for the biliary and pancreatic ducts, which is seen in $75 \%$ of subjects and the length of which is variable $(1-20 \mathrm{~mm})$. The other possibility is a pancreatico-biliary reflux, 
which may occur when pancreatic duct pressure is higher, as suggested by Kune and Sali. ${ }^{7}$ Why should this stone cause perforation of the common bile duct in such an early stage of the disease? It may be explained by excessive friability of an oedematous common bile duct during the acute exacerbation of chronic pancreatitis. The other possibility could be a localized choledochal cyst (Type 1 or 2), the anterior wall of which gave way under minimal rise of common bile duct pressure. However, there was no evidence to support this on the basis of operative findings and his subsequent uneventful course.

The therapeutic attitude, in a sick patient with

\section{References}

1. Scott, J., Summerfield, J.A., Elias, E., Dick, R. \& Sherlock, S. Chronic pancreatitis: a cause of cholestasis. Gut 1977, 18: 196-201.

2. Bradley, E.L. Complications of chronic pancreatitis. Surg Clin North Am 1989, 69: 481-497.

3. Sarles, H. \& Sahel, J. Cholestasis and lesions of the biliary tract in chronic pancreatitis (Progress report). Gut 1978, 19: $851-857$.

4. Stahl, T.J., Allen, M.O., Ansel, H.J. \& Vennes, J.A. Partial biliary obstruction caused by chronic pancreatitis. An appraisal of indications for surgical biliary drainage. Ann Surg 1988, 207: 26-32. bile peritonitis due to perforation of the anterior wall of the common bile duct, should be one of minimal intervention and preservation of the maximum length of common bile duct, while at the same time effectively diverting the bile flow. This can easily be achieved with simple $T$-tube drainage. Although more complicated procedures such as choledocho-jejunostomy Roux-en-Y, with or without, a T-tube may be advocated by some, in an acutely ill patient, the principle of minimal intervention should be followed and efforts should be made to preserve maximum length of the common bile duct. This will help in the management of any future common bile duct stricture.

5. Rai, R.R., Nundy, S., Vashisht, S. \& Tandon, R.K. Chronic calcific pancreatitis: Clinical profile in Northern India. Gastroenterol Jap 1988, 23: 195-200.

6. Balkrishnan, V. Chronic calcific pancreatitis in the tropics. Editorial. Indian J Gastroenterol 1984, 3: 65-67.

7. Kune, G.A. \& Sali, A. Biliary peritonitis. In: Kune, G.A. \& Sali, A. (eds): The Practice of Biliary Surgery. Blackwell Scientific Publications, Oxford, 1980, pp. 317-325. 